\title{
BMJ Open Incidence of depression and depressive symptoms and their predictive factors in community-dwelling older adults: a systematic review and meta-analysis protocol
}

\author{
Lízie Emanuelle Eulálio Brasileiro, ${ }^{1}$ Aílla Lorenna de Medeiros Paiva, ${ }^{1}$ \\ Maria Yasmin Dantas de Medeiros, ${ }^{1}$ Javier Jerez-Roig (1) ,2 \\ Dyego Leandro Bezerra de Souza ${ }^{3}$
}

To cite: Brasileiro LEE, Paiva ALdM, de Medeiros MYD, et al. Incidence of depression and depressive symptoms and their predictive factors in community-dwelling older adults: a systematic review and meta-analysis protocol. BMJ Open 2021;11:e052147. doi:10.1136/ bmjopen-2021-052147

- Prepublication history for this paper is available online To view these files, please visit the journal online (http://dx.doi. org/10.1136/bmjopen-2021052147).

Received 07 April 2021 Accepted 08 July 2021

Check for updates

(C) Author(s) (or their employer(s)) 2021. Re-use permitted under CC BY-NC. No commercial re-use. See rights and permissions. Published by BMJ.

For numbered affiliations see end of article.

Correspondence to Dr Javier Jerez-Roig; javier.jerez@uvic.cat

\section{ABSTRACT}

Introduction Faced with the continuous growth in the number of older people at a global level, some concerns are raised about the way people age. Health conditions such as depressive symptoms and depression have a direct or indirect impact on the quality of life of this population segment. The objective of this study is to verify the incidence of the various presentations of the depressive spectrum in the community-dwelling older population, as well as to analyse the predictive factors. Methods and analysis This systematic review and meta-analysis protocol follows the recommendation of the Preferred Reporting Items for Systematic Reviews and Meta-Analyses Protocols. Searches will be conducted in PubMed, Web of Science, Scopus, Latin-American and Caribbean Literature on Health Sciences, Scientific Electronic Library Online and Cochrane databases, as well as grey literature. The search strategy involves terms related to ageing and the depressive spectrum found in observational studies. There will be no language restriction and the material included will be the ones whose publications took place until December 2020.

Ethics and dissemination Formal ethical approval is not required on this research, since it only aims secondary data. After publishing the results in a scientifically supported journal, our findings may be disseminated to fill in the gaps and guide the production of more effective public policies directed at a more adequate care to the older population at a global level. The search process began in January 2021 and it is expected that all stages of the review will be completed by 30 November 2021. PROSPERO registration number CRD42019121616.

\section{INTRODUCTION}

The number of people aged 65 years and over, according to a United Nations report, ${ }^{1}$ is expected to increase from $9 \%$ in 2019 to $16 \%$ in 2050 . In addition to these projections, the rapid ageing of the population is likely to continue until the middle of the 21 st century, ${ }^{2}$ which entails a greater need for societies to
Strengths and limitations of this study

- To the best of our knowledge, this is the first systematic review and meta-analysis to reveal data on incidence and risk factors of the depressive spectrum in community-dwelling older adults.

- This research adheres to the Preferred Reporting Items for Systematic Reviews and Meta-Analyses guidelines for Protocols.

- Heterogeneous data obtained for the depressive spectrum may limit the performance of quantitative meta-analysis.

- Our choice of not to limit time or language makes the data that will be obtained important from a public health perspective.

adapt. Such demographic changes demand a better dimensioning of the dynamics of ageing, which includes an understanding of the relationship between greater longevity and the qualitative aspect of experiences. Studies show an opposite relationship: the longer one lives the more difficult life experiences can be due to the progressive loss of physical, mental and cognitive integrity. ${ }^{34}$

It is observed a tendency of growth in the prevalence of multimorbidity with the increase of age, ${ }^{5}$ which is related to repercussions in the quality of life and functionality. ${ }^{6}$ Little is known about the relationship between multimorbidity and depression. It has been noticed that people with multimorbidity present a twofold to threefold increase in the chance of having depression when compared with people who do not have $\mathrm{it}^{7}$ and that depression seems to play a central role in the patterns of multimorbidity. ${ }^{8}$

Regarding depression, the $\mathrm{WHO}^{9}$ positions it as the single largest contributor to disability, 
besides its direct relationship with suicide deaths. Depression in the older population is associated with an increased risk of mortality, ${ }^{10}$ along with the impact on functional capacity, fragility ${ }^{11}$ and quality of life, ${ }^{12}{ }^{13}$ being considered as the single largest contributor to non-fatal health loss. ${ }^{9}$ Among the age groups, a peak in the frequency of depression was detected between the age of 55 and 74 years, ${ }^{9}$ which affects $7.5 \%$ of women and $5.5 \%$ of men, worldwide, a percentage that exceeds the other ages.

Clinical characteristics of depression include both psychological symptoms-sadness, lack of energy or decreased ability to feel pleasure-and physical and neurovegetative symptoms-such as fatigue, changes in sleep and appetite. ${ }^{14}$ This range of symptoms is associated with neurochemical changes of a polygenic nature, ${ }^{15}$ as well as more subjective factors that occur throughout life, such as bereavements and losses, individual coping capacity, social support and loneliness. ${ }^{916} 17$ This heterogeneous profile of the clinical manifestation of depression makes its study complex, especially when so many forms of presentation can occur.

When discussing depression in the scientific literature, particularly when conducting epidemiological studies, depression can take on several different presentations. The way depression has been researched entails a great variability in prevalence and incidence data, which makes the comprehension of the presentations important to be addressed. This variability in general is due to methodological differences: (1) how the depressive spectrum can be researched; (2) the housing profile of the older population.

In the first difference, the depressive spectrum is understood as the entire set of depressive categories, from the mildest depressive symptoms to the most severe, such as major depression. ${ }^{18} 19$ There are two ways to research the depressive spectrum, either by categorisation or by dimensional assessment. The categorisation is determined by the American Psychiatric Association, in its 5th edition of the Diagnostic and Statistical Manual of Mental Disorders, Fifth Edition (DSM-5), ${ }^{14}$ or by the WHO, by the International Code of Diseases 10th edition (ICD-10) ${ }^{20}$ in the so-called depressive disorders. For dimensionality assessment, depression scales quantify and establish a cutoff point, revealing what is known as clinically significant depressive symptoms (CSDS). This type of assessment of depression seems to have a good representation in its syndromic understanding. ${ }^{21}$
It is important to emphasise that both ways of assessing depression are relevant. Even when evaluating the CSDS, the so-called 'subclinical' depression, there is a tendency to chronicity and recurrence, ${ }^{11}{ }^{22}$ which entails the psychosocial repercussions. ${ }^{23}$ In this regard, early detection should be a focus at the primary healthcare level, with a view to the early establishment of interventions. ${ }^{23}$

As for the housing profile of the older population, it is known that those who live in their own homes have better quality of life indexes ${ }^{24}$ and a better level of functionality than the institutionalised ${ }^{25} 26$ or hospitalised ${ }^{27}$ older adults. Therefore, a systematic review focusing on community-dwelling older adults intends to address a preventive perspective, especially when involving studies of incidence and assessment of predictive factors of depression.

Notwithstanding the review research carried out in 2003, which sought to evaluate risk factors for depression, ${ }^{28}$ there are no updated reviews or meta-analysis whose population focus is community-dwelling adults aged 60 years or over, that particularly bring incidence rates or reveal predictive factors. We hypothesise that sociodemographic factors, health conditions, social support, functional disability and some contextual factors may predict the depressive spectrum in communitydwelling older adults.

The objective of this systematic review will be to ascertain the incidence in the various presentations of the depressive spectrum in the community-dwelling older population, as well as to analyse the predictive factors.

\section{METHODS AND ANALYSIS \\ Study design and eligibility criteria}

This research is a systematic review that includes observational studies whose research questions should aim to assess the incidence of depression within its depressive spectrum and its predictive factors. The protocol was registered with the International Prospective Register of Systematic Reviews (PROSPERO).

As recommended by Preferred Reporting Items for Systematic Reviews and Meta-Analyses (PRISMA), ${ }^{29}$ systematic reviews should be guided by the acronym PICO, representing the participants, intervention (in our case, we replace it by exposure variables), comparators and outcomes, which can be seen summarised in table 1 .

\begin{tabular}{lll}
\hline \multicolumn{2}{l}{ Table 1} & PICO description \\
\hline Abbreviation & Meaning & Characteristics \\
\hline P & Participants & Community-dwelling adults $\geq 60$ years old. \\
I & $\begin{array}{l}\text { Intervention } \\
\text { (exposure) }\end{array}$ & $\begin{array}{l}\text { Sociodemographic factors, health conditions, social support, functional disability and } \\
\text { contextual factors. }\end{array}$ \\
C & Comparators & Group without the exposure variables. \\
O & Outcomes & Prevalence/incidence data of depression/clinically significant depressive symptoms. \\
\hline
\end{tabular}


As defined by the WHO, ${ }^{30}$ individuals older than 65 years are considered old in developed countries, while the cut-off point decreases to 60 years old in developing countries. Thus, we will include articles whose population focus should be exclusively older adults, of both sexes, community-dwelling adults aged 60 years or older, that is, living in a home or residence, even if they live in houses with other older people or receive specialised care, and therefore are not institutionalised or hospitalised.

We will include the primary studies that deal with depression or clinically significant depressive symptoms. To embrace the nomenclature used for depressive spectrum, studies that research major depressive episode, major depressive disorder, unipolar depression, dysthymia, minor depression, subclinical depression and CSDS will be included. This variable, related to the depressive spectrum, will be treated as the dependent variable or the primary outcome. Studies that include bipolar depression or depression with psychotic symptoms will be excluded.

The evaluation of the predictive factors of depression or depressive symptoms will be considered the independent variable and have been summarised in table 1 . These variables can be further broken down into others and will depend on the articles included at the end of the systematic review. The factors hypothesised here to be predictive of the depressive spectrum have been studied over time. For example, female gender and age group within the older adults population among the sociodemographic variables, comorbidities/multimorbidities or sphincter alterations as health conditions, lack of ties with friends, family or loneliness as social support, losses or bereavements as contextual factors and, impairment in functionality as functional disability, which translates the level of dependence. ${ }^{9162531}$

Regarding language, the team will include all studies that meet these eligibility criteria, with no initial restriction for languages considering that it could compromise the more global intent of this systematic review protocol. Therefore, for articles whose languages are not within the team's domain, the first attempt would be to look for translators whose material is reliable. If this is not possible, the languages will include articles in English, Spanish, Portuguese and French, being recognised as a limitation of the systematic review.

Studies that include blood, molecular or chemical element measurements will be excluded. Studies investigating clinical populations in specific outpatient treatment or presenting specific diseases will also be excluded.

\section{Outcome measures}

The primary outcome can be subdivided into: (1) Incidence of depression, by means of measures of incidence rate or cumulative incidence of a major depressive episode or of clinically significant depressive symptoms. If scales are used to measure the depressive variable, they must be validated, and the final values can be displayed in a dichotomous or continuous manner. Self-report measures of depression will not be accepted; (2) Predictive factors of the depressive spectrum may be arranged in dichotomous values, to be analysed by measures of association with $95 \%$ CI.

Furthermore, as a secondary outcome, longitudinal articles will be searched, whose objectives seek to evaluate the trajectory of the depressive spectrum, through the follow-up of symptom severity (measured with scales) or depression, by means of the DSM-5 and ICD-10, and its associated variables.

\section{Information source and search strategy}

Six health-related databases were used : PubMed, Web of Science, Scopus, Latin-American and Caribbean Literature on Health Sciences, Scientific Electronic Library Online and Cochrane. In addition to these bases, materials from the grey literature will be used, represented by repositories of monographs or theses, publications in Congresses and Google Scholar.

Despite the possibility of using filters tools in advanced searches in the various databases, the authors have decided not to include in the search strategy step the filters of document types, languages and types of studies, since the search was intended to be exhaustive. We have established the best possible search strategy so that more relevant studies could emerge within the theme, using several combinations of descriptors and key words in the equation. Regarding the use of the associated terms 'prevalence study' OR 'incidence study', compared with the search strategy that used only 'incidence study', no considerable difference was observed between the results of the different searches in the quantity of articles per database. Thus, we have opted to use the first association, anticipating that the differentiation between crosssectional and longitudinal studies would be left to be done in the peer review phase.

Considering the search strategy for this study, literature search strategies were developed using medical subject headings and text words related to the population, depressive spectrum and the type of study, forming an equation that respected the search form and technique in each base, according to table 2 .

In the spirit of including as much relevant material as possible, studies that answer the research question and are listed in their references will be included in the screening. For papers that may not be found in their complete form, we will establish communication with each lead author so

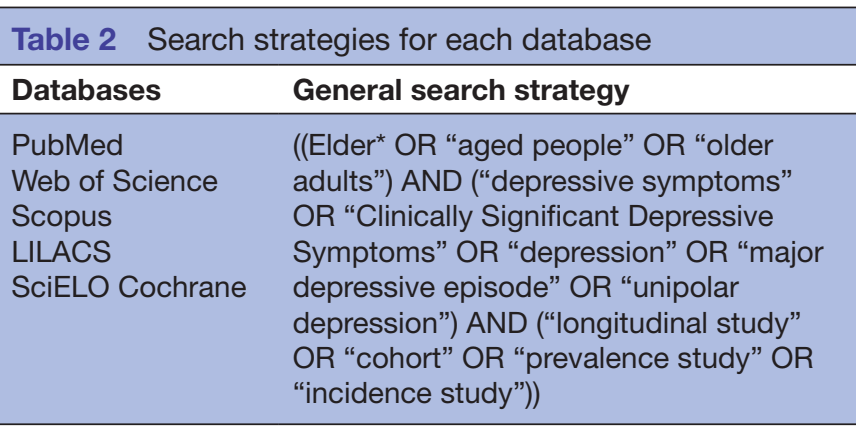


that the material can be properly evaluated according to the eligibility criteria.

The grey literature can be accessed by unfiltered search in some databases through conference proceedings, Google Scholar, monograph or thesis repositories, grey literature search websites such as the Canadian Agency for Health Technology Assessment ${ }^{32}$ and the references of the included studies. The last date for collecting the material was on 31 December 2020.

\section{Review and selection process of studies}

A total of seven researchers will participate in this review and the whole process will be accompanied by face-toface or virtual meetings, weekly or biweekly, for training, adjustments, as well as to dispel disagreements.

After using the aforementioned search strategy, Microsoft Excel will be used in order to make it possible to count the studies per base and remove duplicates. The selection of these studies will begin by peer review and independent reading (two members of the group and a third participant will be responsible for dispelling any disagreements found and maintaining the alignment of the eligibility criteria). The selection phase will be consisted of two moments sequentially: (1) reading the titles and abstracts and (2) reading the full text, counting the number of studies included for the next phase and those excluded with their justifications. These results will be demonstrated at the time of publication of the systematic review using the flowchart recommended by PRISMA. ${ }^{29}$

\section{Data extraction}

After selecting the studies, data extraction will occur in a paired and independent approach, using a third component to assess disagreements. The data to be extracted will be guided by the purpose of this study, associated with the eligibility criteria. Therefore, it should include data on the country from which the study was developed, population type and location, study design, inclusion and exclusion criteria, observation time, sociodemographic characteristics at the first wave, predictive factors and outcome variable characteristics, such as the nature of the variable, instrument and values. These data will be arranged in a table, using Microsoft Excel.

\section{Evaluation of quality of the studies}

This phase of the study is based on the evaluation of quality of the studies included by peer review, and thus, the analysis of the risk of bias. The quality assessment will be guided by the evaluation of the representativeness of the sampling, the form of measurement, the comparison of exposed and unexposed, the observation of values related to the outcomes and the control of confounding factors.

The assessment of quality will be evaluated by the chosen tool called Quality Assessment Tool for Observational Cohort and Cross-Sectional Studies. ${ }^{33}$ It consists of 14 items, to be also evaluated in a peer review, with a third member to dispel disagreements with 'yes', 'no' or 'other' (which includes 'cannot determine', 'not applicable' or 'not reported').

\section{Biases and heterogeneity}

After the quantitative synthesis of the meta-analysis is done, we will evaluate heterogeneity, due to the variety of instruments customarily used to assess the outcome of the depressive spectrum, besides other possible differences in the studies analysed. The $\mathrm{I}^{2}$ test will be used, being admitted as heterogeneous if $\mathrm{I}^{2}$ values are above $50 \%$. In case of heterogeneity, a subgroup analysis will be performed from the selection of certain included studies whose population or data of older people differ, such as countries separated into categories by socioeconomic differences according to the United Nations classification and aggregation methodology.

Graphical techniques such as funnel charts or statistical techniques (depending on the nature of the variable) will be employed to evaluate publication bias.

\section{Summary data}

In this stage, incidence data of the depressive spectrum will be presented, as well as the risk factors. This section corresponds to the meta-analysis itself, and if it is not possible, a narrative and qualitative synthesis will be made.

Different meta-analyses will be performed for the quantitative data incidence studies and according to the variability of the depressive spectrum, using aggregate data. For each study included in the meta-analysis, we will use the inverse-variance weighting method. It will be critical to assess the impact of heterogeneity, especially since there are so many ways to evaluate the depressive spectrum.

The data will be analysed by the software R Project and displayed in tables, which will contain the measures of frequency and effect, whether continuous or dichotomised, with exposure of the relative risk, OR or mean differences in case the depressive variable is presented in a continuous form. Furthermore, forest plot graphics may be presented.

\section{Reliance on the evidence found}

The assessment of the quality of the evidence that will be included should be addressed by The Grading of Recommendations Assessment, Development and Evaluation, which makes it possible to classify into reliable levels, evaluating whether the effect estimates are close to reality.

\section{Patient and public involvement \\ No patient involved.}

\section{ETHICS AND DISSEMINATION}

Formal ethical approval is not required on this research, since published studies with non-identifiable data will be used. It is known that a systematic review and meta-analysis follow a series of steps, respecting methodological rigour and synthesising scientific evidence reliably. After publication of the final report of this material in a scientifically 
supported journal, the results can provide a global picture of the depressive spectrum in the community-dwelling older adults population, highlighting similarities between the different locations, as well as the differences. The epidemiological aspects found may fill current gaps and encourage the development of public policies focused on the health of older adults, especially for the group with lower vulnerability. Moreover, with the dissemination of the results, more studies can be done with the intention of understanding the impact of the insertion of new tools in healthcare for older adults.

Amendments: After approval of the protocol, any important amendments will be documented in the final publication; and if necessary, these amendments will be registered with PROSPERO.

\section{Author affiliations}

${ }^{1}$ Multicampi School of Medical Sciences, Federal University of Rio Grande do Norte, Caicó, RN, Brazil

${ }^{2}$ Research group on Methodology, Methods, Models and Outcomes of Health and Social Sciences $\left(\mathrm{M}_{3} \mathrm{O}\right)$. Faculty of Health Sciences and Welfare. Centre for Health and Social Care Research (CESS), University of Vic-Central University of Catalonia (UVic-UCC), Vic, Spain

${ }^{3}$ Postgraduate Programme in Collective Health, Federal University of Rio Grande do Norte, Natal, RN, Brazil

\section{Twitter Javier Jerez-Roig @javierjerezroig}

Acknowledgements The authors wish to acknowledge the support of the National Council for Scientific and Technological Development (CNPq, Research Productivity grants 308168/2020-8)

Contributors JJ-R is the guarantor. Conceptualisation: LEEB, DLBdS and JJ-R. Data analysis: LEEB, DLBdS and JJ-R. Methodology and project administration: LEEB, DLBdS, JJ-R, ALdMP and MYDdM. Reading and final revision of the text; LEEB, DLBdS, JJ-R, ALdMP and MYDdM. Research: All. Writing of the scientific paper: LEEB, DLBdS, JJ-R, ALdMP and MYDdM.

Funding This study was funded in part by the Coordenação de Aperfeiçoamento de Pessoal de Nível Superior-Brasil (CAPES)—Finance Code 001. CAPES will only be responsible for financing the publication fee in the journal, and therefore is not involved in any other aspect of the project, such as the design of the project's protocol, analysis plan, collection and analyses.

Competing interests None declared.

Patient and public involvement Patients and/or the public were not involved in the design, or conduct, or reporting, or dissemination plans of this research.

Patient consent for publication Not required.

Provenance and peer review Not commissioned; externally peer reviewed.

Open access This is an open access article distributed in accordance with the Creative Commons Attribution Non Commercial (CC BY-NC 4.0) license, which permits others to distribute, remix, adapt, build upon this work non-commercially, and license their derivative works on different terms, provided the original work is properly cited, appropriate credit is given, any changes made indicated, and the use is non-commercial. See: http://creativecommons.org/licenses/by-nc/4.0/.

\section{ORCID iD}

Javier Jerez-Roig http://orcid.org/0000-0002-1968-4452

\section{REFERENCES}

1 UN. World population prospects 2019. New York: United Nations Population Division, 2019. https://population.un.org/wpp/Download/ Standard/Population/

2 Lutz W, Sanderson W, Scherbov S. The coming acceleration of global population ageing. Nature 2008;451:716-9.

3 Beard JR, Officer A, de Carvalho IA, et al. The world report on ageing and health: a policy framework for healthy ageing. The Lancet 2016;387:2145-54.
4 Chang AY, Skirbekk VF, Tyrovolas S, et al. Measuring population ageing: an analysis of the global burden of disease study 2017. Lancet Public Health 2019;4:e159-67.

5 Souza DLB, Oliveras-Fabregas A, Minobes-Molina E, et al. Trends of multimorbidity in 15 European countries: a population-based study in community-dwelling adults aged 50 and over. BMC Public Health $2021 ; 21: 76$

6 Marengoni A, Angleman S, Melis R, et al. Aging with multimorbidity: a systematic review of the literature. Ageing Res Rev 2011;10:430-9.

7 Read JR, Sharpe L, Modini M, et al. Multimorbidity and depression: a systematic review and meta-analysis. J Affect Disord 2017;221:36-46.

8 Birk JL, Kronish IM, Moise N, et al. Depression and multimorbidity: considering temporal characteristics of the associations between depression and multiple chronic diseases. Health Psychology 2019;38:802-11.

9 InWHO. Depression and other common mental disorders: global health estimates. Services DP, 2017.

10 Ho CS, Jin A, Nyunt MSZ, et al. Mortality rates in major and subthreshold depression: 10-year follow-up of a Singaporean population cohort of older adults. Postgrad Med 2016;128:642-7.

11 Collard RM, Arts MHL, Schene AH, et al. The impact of frailty on depressive disorder in later life: findings from the Netherlands study of depression in older persons. Eur Psychiatry 2017;43:66-72.

12 Rong J, Chen G, Wang X, et al. Correlation between depressive symptoms and quality of life, and associated factors for depressive symptoms among rural elderly in Anhui, China. Clin Interv Aging 2019;14:1901-10.

13 Sivertsen H, Bjørkløf GH, Engedal K, et al. Depression and quality of life in older persons: a review. Dement Geriatr Cogn Disord 2015;40:311-39.

14 APA. Diagnostic and statistical manual of mental disorders- DSM-5. 5th ed. American Psychiatric Association, 2013: 155-88.

15 Howard DM, Adams MJ, Clarke T-K, et al. Genome-Wide metaanalysis of depression identifies 102 independent variants and highlights the importance of the prefrontal brain regions. Nat Neurosci 2019;22:343-52.

16 Hsueh Y-C, Chen C-Y, Hsiao Y-C, et al. A longitudinal, cross-lagged panel analysis of loneliness and depression among communitybased older adults. J Elder Abuse Negl 2019;31:281-93.

17 do Nascimento KKF, Pereira KS, Firmo JOA, et al. Predictors of incidence of clinically significant depressive symptoms in the elderly: 10 -year follow-up study of the Bambui cohort study of aging. Int $J$ Geriatr Psychiatry 2015;30:1171-6.

18 Fiske A, Wetherell JL, Gatz M. Depression in older adults. Annu Rev Clin Psychol 2009;5:363-89.

19 Luppa M, Sikorski C, Luck T, et al. Age- and gender-specific prevalence of depression in latest-life - systematic review and metaanalysis. J Affect Disord 2012;136:212-21.

20 WHO. The ICD-10 classification of mental and behavioural disorders clinical descriptions and diagnostic guidelines. 10th ed. World Health Organization, 1992.

21 Eulálio MdoC, Andrade TFde, Melo RLPde, et al. [Latent structure of depression in the elderly: a taxometric analysis]. Cad Saude Publica 2015;31:555-64.

22 Beekman AT, Geerlings SW, Deeg DJ. The natural history of late-life depression: a 6-year prospective study in the community. Arch Gen Psychiatry 2002;59:605-11.

23 Ayuso-Mateos JL, Nuevo R, Verdes E, et al. From depressive symptoms to depressive disorders: the relevance of thresholds. $\mathrm{Br} \mathrm{J}$ Psychiatry 2010;196:365-71.

24 Dias E, Pais-Ribeiro J. Qualidade de vida: comparação entre OS idosos Na comunidade E institucionalizados. Revista KairósGerontologia 2018;21:37-54.

25 Jerez-Roig J, de Medeiros JF, do Nascimento Moreira Fidélis K, et al Activity limitations in Brazilian institutionalized older adults. $J$ Geriatr Phys Ther 2017;40:214-22.

26 Lima-Costa MF, Matos DL, Camargos VP, et al. [10-year trends in the health of Brazilian elderly: evidence from the National Household Sample Survey (PNAD 1998, 2003, 2008)]. Cien Saude Colet 2011;16:3689-96.

27 Melo-Silva AM, Mambrini JVM, Souza-Junior PRB. Hospitalizações entre adultos MAIS velhos: resultados do ELSI-Brasil. Revista Saúde Pública 2018;52:3s.

28 Cole MG, Dendukuri N. Risk factors for depression among elderly community subjects: a systematic review and meta-analysis. AJP 2003;160:1147-56.

29 Moher D, Liberati A, Tetzlaff J, et al. Preferred reporting items for systematic reviews and meta-analyses: the PRISMA statement. International Journal of Surgery 2010;8:336-41. 
30 WHO. The uses of epidemiology in the study of the elderly. Report of a who scientific group on the epidemiology of aging. World Health Organ Tech Rep Ser. Geneva 1984:1-84.

31 Bezerra de Souza DL, Oliveras-Fabregas A, Espelt A, et al. Multimorbidity and its associated factors among adults aged 50 and over: a cross-sectional study in 17 European countries. PLoS One 2021;16:e0246623.
32 CADTH. Canadian agency for drugs and technologies in health, 2019. Available: https://www.cadth.ca/resources/finding-evidence/ grey-matters [Accessed December 20 2020].

$33 \mathrm{NIH}$. Study Quality Assessment Tools. Available: https://www.nhlbi. nih.gov/health-topics/study-quality-assessment-tools [Accessed 5 February 2021]. 